\title{
THE SPACE OF CLASS $\alpha$ BAIRE FUNCTIONS
}

\author{
BY J. E. JAYNE
}

Communicated by Jacob Feldman, December 6, 1973

\begin{abstract}
Let $X, Y$ be compact Hausdorff spaces and $B_{\alpha}^{*}(X)$, $B_{\beta}^{*}(Y), 0 \leqslant \alpha, \beta \leqslant \Omega$ (the first uncountable ordinal), the associated Banach spaces of bounded real-valued Baire functions of classes $\alpha$ and $\beta$. If $B_{\alpha}^{*}(X) \neq B_{\beta}^{*}(X)$ (which is the case if $\alpha \neq \beta$ and $X$ is not dispersed), then $B_{\alpha}^{*}(X)$ is neither linearly isometric to $B_{\beta}^{*}(Y)$ nor equivalent to $B_{\beta}^{*}(Y)$ in several other ways. $B_{\Omega}^{*}(X)$ is linearly isometric to $B_{\Omega}^{*}(Y)$ if and only if $X$ is Baire isomorphic to $Y$. For $1 \leqslant \alpha<\Omega$ the maximal ideal space of $B_{\alpha}^{*}(X)$ for a nondispersed compact space $X$ is not an $F$-space.
\end{abstract}

1. Let $X$ be a compact (more generally, completely regular) Hausdorff space and $C(X)$ the space of continuous real-valued functions on $X$. Let $B_{0}(X)=C(X)$, and inductively define $B_{\alpha}(X)$ for each ordinal $\alpha \leqslant \Omega(\Omega$ denotes the first uncountable ordinal) to be the space of pointwise limits of sequences of functions in $\bigcup_{\xi<\alpha} B_{\xi}(X)$. Let $B_{\alpha}^{*}(X)$ be the space of bounded functions contained in $B_{\alpha}(X)$. With the pointwise operations $B_{\alpha}(X)$ and $B_{\alpha}^{*}(X)$ are lattice-ordered algebras. With the supremum norm $B_{\alpha}^{*}(X)$ is a Banach algebra (see $[4, \S 41]$ ).

The Baire sets of $X$ of multiplicative class $\alpha$, denoted by $Z_{\alpha}(X)$, are defined to be the zero sets of functions in $B_{\alpha}^{*}(X)$. Those of additive class $\alpha$, denoted by $C Z_{\alpha}(X)$, are defined as the complements of sets in $Z_{\alpha}(X)$.

Finally, those of ambiguous class $\alpha$, denoted by $A_{\alpha}(X)$, are the sets which are simultaneously in $Z_{\alpha}(X)$ and $C Z_{\alpha}(X)$. With the set-theoretic operations of union and intersection, $A_{\alpha}(X)$ is a Boolean algebra for each $\alpha \leqslant \Omega$. The sets of exactly ambiguous class $\alpha$, denoted by $E A_{\alpha}(X)$, are those in $A_{\alpha}(X) \backslash \bigcup_{\xi<\alpha} A_{\xi}(X)$. The sets of exactly additive and exactly multiplicative class $\alpha$ are defined analogously. The class of all Baire subsets of $X$ is $Z_{\Omega}(X)$.

AMS (MOS) subject classifications (1970). Primary 06A65, 26A21, 28A05, 46E15, 46E30, 46J10, 54C50, 54H05; Secondary 04A15.

Key words and phrases. Banach space, Baire function, Baire class. 
A topological space is called realcompact if it is homeomorphic to a closed subset of a product of real lines.

THEOREM 1. If $X$ and $Y$ are compact (more generally, realcompact) spaces, then every Boolean algebra isomorphism $f$ of $A_{\alpha}(X)$ onto $A_{\beta}(Y)$, $1 \leqslant \alpha, \beta \leqslant \Omega$, is induced by a point map $\phi$ of $X$ onto $Y$; that is, there exists a one-to-one map $\phi$ of $X$ onto $Y$ such that $\phi[B]=f(B)$ for each $B \in A_{\alpha}(X)$.

Proof outline. Consider the compact set, denoted by $X_{\alpha}$, of nonzero multiplicative linear functionals on $B_{\alpha}^{*}(X)$ with the weak star topology. It follows from the fact that for each pair of disjoint sets $B_{1}, B_{2} \in Z_{\alpha}(X)$, there is an $A \in A_{\alpha}(X)$ with $B_{1} \subseteq A \subseteq X \backslash B_{2}$, that $X_{\alpha}$ has a base of clopen (closed and open) sets. Since the Boolean algebra of clopen sets of $X$ is isomorphic to $A_{\alpha}(X)$, the Stone space of $A_{\alpha}(X)$ is homeomorphic to $X_{\alpha}$.

The canonical embedding of $X$ into $X_{\alpha}$ which assigns a point in $X$ to the evaluation functional at that point maps $X$ onto a dense subset of $X_{\alpha}$. The induced topology on $X$ from $X_{\alpha}$ is discrete if and only if every point in $X$ is a $G_{\delta}$. The space $X_{\alpha}$ may thus be considered as a compactification of $X$ with the topology having $Z_{0}(X)$ as a base. From this point of view, each $f \in B_{\alpha}^{*}(X)$ has a unique extension to a $\hat{f} \in C\left(X_{\alpha}\right)$, and the map $\Phi: B_{\alpha}^{*}(X) \rightarrow C\left(X_{\alpha}\right)$ defined by $\Phi(f)=\hat{f}$ is an algebra isomorphism onto $C\left(X_{\alpha}\right)$. Details concerning the space $X_{\alpha}$ are contained in [5].

A filter $F$ of sets in $A_{\alpha}(X)\left(Z_{\alpha}(X)\right)$ is said to have the CIP (countable intersection property) if for each countable family $\left\{C_{n}\right\} \subseteq F$ there is a $C \in A_{\alpha}(X)$ (respectively $Z_{\alpha}(X)$ ) such that $C \subseteq \bigcap_{n=1}^{\infty} C_{n}$. A filter $F$ in $A_{\alpha}(X)\left(Z_{\alpha}(X)\right)$ is said to be fixed if $\bigcap\{F: F \in F\} \neq \varnothing$.

For any completely regular spaces $X$ and $Y$, if $f$ is a Boolean algebra isomorphism of $A_{\alpha}(X)$ onto $A_{\beta}(Y)$ and $M$ is a maximal filter in $A_{\alpha}(X)$ with the CIP, then $f[M]$ is a maximal filter in $A_{\beta}(Y)$ with the CIP.

If $X$ is realcompact, then every maximal filter with the CIP in $A_{\alpha}(X)$, $\alpha \geqslant 1$, is fixed. To see this let $M \subseteq A_{\alpha}(X)$ be a maximal filter with the CIP. Then, since each element of $Z_{\alpha}(X)$ is the countable intersection of elements in $A_{\alpha}(X), M_{\delta}$ (the family of countable intersections of sets in $M$ ) is a maximal filter with the CIP in $Z_{\alpha}(X)$. Thus, since $X$ is realcompact and each set in $Z_{\alpha}(X)$ is obtainable from $Z_{0}(X)$ by Souslin's operation $(A)$, $M_{\delta}$ is fixed. Thus $M$ is fixed. Here we have used the following set-theoretic result due to Z. Frolik [2]: Let $H_{1}$ and $H_{2}$ be families of subsets of a set 
$X$ which are closed under countable intersections, and let $M\left(H_{i}\right), i=1,2$, denote the set of free maximal filters in $H_{i}$ with the CIP. If $H_{1} \subseteq H_{2}$ and every $H \in H_{2}$ is a Souslin- $H_{1}$ set (that is, can be represented in the form

$$
H=\bigcup_{i_{1}, i_{2}, \cdots} \bigcap_{n=1}^{\infty} H_{i_{1}, \cdots, i_{n}}, \quad H_{i_{1}, \cdots, i_{n}} \in H_{1}
$$

where the union is over all sequences of positive integers $\left.\left(i_{1}, i_{2}, \cdots\right)\right)$, then the map $M \rightarrow M \cap H_{1}, M \in M\left(H_{2}\right)$ is one-to-one onto $M\left(H_{1}\right)$.

From this it follows that if $X$ and $Y$ are realcompact, $\alpha, \beta \geqslant 1$, and $f$ is a Boolean algebra isomorphism of $A_{\alpha}(X)$ onto $A_{\beta}(Y)$, then the induced homeomorphism $\phi$ of their Stone spaces, namely $X_{\alpha}$ and $Y_{\beta}$, maps $X$ onto $Y$; that is, $\phi[X]=Y$. Also for each $B \in A_{\alpha}(X), \phi[B]=f(B)$. This completes the proof.

2. Let $X$ and $Y$ be completely regular spaces. A Baire isomorphism of class $(\alpha, \beta ; \gamma, \delta)$ of $X$ onto $Y$ is a one-to-one map $f$ of $X$ onto $Y$ such that

$$
f\left[Z_{\alpha}(X)\right] \subseteq Z_{\beta}(Y) \text { and } f^{-1}\left[Z_{\delta}(Y)\right] \subseteq Z_{\gamma}(X)
$$

THEOREM 2. If $X$ and $Y$ are compact (more generally, realcompact) spaces and $\alpha, \beta \geqslant 1$, then the following are equivalent:

(1) There exists a Baire isomorphism of class $(\alpha, \beta ; \alpha, \beta)$ of $X$ onto $Y$.

(2) $B_{\alpha}^{*}(X)$ is linearly isometric to $B_{\beta}^{*}(Y)$.

(3), (4), (5), (6) $B_{\alpha}^{*}(X)$ is isometric (ring, lattice, multiplicative semigroup isomorphic) to $B_{\beta}^{*}(Y)$.

(6), (7), (8), (9) $B_{\alpha}(X)$ is ring (lattice, multiplicative semigroup) isomorphic to $B_{\beta}(Y)$.

Proof outline. (2) $\Rightarrow(1)$. Since $B_{\alpha}^{*}(X)$ and $B_{\beta}^{*}(Y)$ are linearly isometric to $C\left(X_{\alpha}\right)$ and $C\left(Y_{\beta}\right)$ respectively, $X_{\alpha}$ and $Y_{\beta}$ are homeomorphic. By Theorem 1 such a homeomorphism induces a Baire isomorphism of class $(\alpha, \beta ; \alpha, \beta)$ of $X$ onto $Y$.

All of the other nontrivial implications follow similarly.

REMARK. Since for a completely regular space $X$ every $f \in B_{\alpha}^{*}(X)$ $\left(B_{\alpha}(X)\right)$ has a unique extension to a $\hat{f} \in B_{\alpha}^{*}(\nu X)\left(B_{\alpha}(X)\right)$, where $v X$ denotes the Hewitt realcompactification of $X[6]$, it follows from Theorem 2 that for completely regular spaces $X$ and $Y$, parts (2) through (9) of Theorem 2 are 
equivalent, and these are equivalent to the existence of a Baire isomorphism of class $(\alpha, \beta ; \alpha, \beta)$ of $\nu X$ onto $\nu Y$. More generally yet, Theorems 1 and 2 may be phrased in terms of zero-set spaces and suitably defined 0-dimensional zero-set spaces, their realcompactifications, and their associated function spaces (see [3]).

3. Recently F. Dashiell [1] has shown that if $X$ is an uncountable compact metric space, then for $\alpha \neq \beta, B_{\alpha}^{*}(X)$ is not linearly isometric to $B_{\beta}^{*}(X)$, which may be thought of as strengthening the classical result that for $\alpha<\beta$, $B_{\alpha}^{*}(X)$ is a proper subspace of $B_{\beta}^{*}(X)$.

A compact space is called dispersed if it contains no nonempty perfect subsets. It is known that a compact space $X$ contains a nonempty perfect subset if and only if for each $\alpha<\Omega, B_{\alpha}^{*}(X)$ is a proper subspace of $B_{\alpha+1}^{*}(X)$, and if and only if $B_{2}^{*}(X) \backslash B_{1}^{*}(X) \neq \varnothing$ (see [5] and [6]). Part of this also follows from the next theorem, since a nondispersed compact space admits a continuous map onto the unit interval.

THEOREM 3. If $X$ is a compact space, $\alpha \geqslant 0, f$ a continuous realvalued map on $X$, and $B \in E A_{\alpha}(f[X])$, then $f^{-1}[B] \in E A_{\alpha}(X)$. The same holds for exactly additive and exactly multiplicative classes.

THEOREM 4. If $f$ is a continuous map of a compact space $X$ onto a compact space $Y$, then for $\alpha=0,1,2$ or $\alpha \geqslant \omega_{0}, B \in E A_{\alpha}(Y)$ implies that $f^{-1}[B] \in E A_{\alpha}(X)$, and for $2<\alpha<\omega_{0}, B \in A_{\alpha}(Y)$ implies that $f^{-1}[B] \in E A_{\alpha-1}(X) \cup E A_{\alpha}(X)$. The same holds for exactly additive and exactly multiplicative classes.

THEOREM 5. (1) Let $X$ and $Y$ be compact spaces and suppose that either $X$ or $Y$ is not dispersed. For $0 \leqslant \alpha<\beta \leqslant \Omega, B_{\alpha}^{*}(X)$ is not linearly isometric to $B_{\beta}^{*}(Y)$.

(2) If $X$ and $Y$ are infinite dispersed compact spaces, then $B_{0}^{*}(X)$ is not linearly isometric to $B_{1}^{*}(Y)$. (Note that $B_{1}^{*}(X)=B_{2}^{*}(X)$.)

Proof outline. (1) Suppose $\alpha<\beta$, that $X$ is not dispersed, and that $B_{\alpha}^{*}(X)$ is linearly isometric to $B_{\beta}^{*}(Y)$. Then by Theorem 2 there is a Baire isomorphism $\phi$ of $Y$ onto $X$ of class $(\beta, \alpha ; \beta, \alpha)$. Thus there is a ring isomorphism $\Phi$ of $B_{\Omega}(X)$ onto $B_{\Omega}(Y)$ such that $\Phi\left[B_{\alpha}(X)\right]=B_{\beta}(Y)$ defined by $\Phi(h)(y)=h(\phi(y))$ for all $h \in B_{\alpha}(X)$ and $y \in Y$. Let $f: X$ $\rightarrow[0,1]$ be a continuous map onto the unit interval. Let $\left\{g_{n}: n=1,2\right.$, $\cdots\} \subseteq C(Y)$ be such that $\Phi(f)$ is contained in the smallest class of functions 
containing $\left\{g_{n}: n=1,2, \cdots\right\}$ and closed under pointwise sequential limits.

Consider the map $\Psi: Y \rightarrow \mathbf{R}^{N}$ defined by $\Psi(y)=\left(g_{1}(y), g_{2}(y), \cdots\right)$. Then the Baire isomorphism $\phi$ of $Y$ onto $X$ induces a Baire measurable map $\widetilde{\phi}$ of $\Psi[Y]$ onto $[0,1]$. There is a Cantor set $C \subseteq \Psi[Y]$ such that $\widetilde{\phi}$ restricted to $C$ is a homeomorphism [7, p. 444], since $\widetilde{\phi}$ is continuous apart from a set of first category [7, p. 400]. Thus there is a set $B \in$ $E A_{\beta}(\widetilde{\phi}[C]) \subseteq E A_{\beta}([0,1])$. By Theorem $3, \Psi^{-1}\left[\widetilde{\phi}^{-1}[B]\right] \in E A_{\beta}(Y)$ and $f^{-1}[B] \in E A_{\beta}(X)$.

This implies that the characteristic function

$$
\chi_{\Psi^{-1}\left[\tilde{\phi}^{-1}[B]\right]} \in B_{\beta}^{*}(Y) \backslash \bigcup_{\xi<\beta} B_{\xi}^{*}(Y)
$$

But since $\Phi^{-1}\left(\chi_{\Psi^{-1}\left[\tilde{\phi}^{-1}[B]\right]}\right)=\chi_{f^{-1}[B]}$, it follows that $\chi_{f^{-1}[B]} \in B_{\alpha}^{*}(X)$. This contradiction completes the proof.

(2) This follows from the fact that $X_{1}$ and $Y_{1}$ contain nonempty compact perfect subsets and $X$ and $Y$ do not.

4. A topological space $X$ is called an $F$-space if for each disjoint pair $C_{1}, C_{2} \in C Z_{0}(X)$ there is a disjoint pair $Z_{1}, Z_{2} \in Z_{0}(X)$ such that $C_{1} \subseteq Z_{1}$ and $C_{2} \subseteq Z_{2}$.

LEMmA. Let $X$ be any topological space and $\alpha \geqslant 1$. If $Z_{1}, Z_{2} \in$ $Z_{\alpha}(X)$ are disjoint, then' there is a set $A \in A_{\alpha}(X)$ such that $Z_{1} \subseteq A \subseteq$ $X \backslash Z_{2}$.

THEOREM 6. If $X$ is a nondispersed compact space and $1 \leqslant \alpha<\Omega$, then there exist disjoint sets $C_{1}, C_{2} \in C Z_{\alpha}(X)$ such that there does not exist a set $A \in A_{\alpha}(X)$ with $C_{1} \subseteq A \subseteq X \backslash C_{2}$. Consequently, $X_{\alpha}$, the Stone space of $A_{\alpha}(X)$, is not an $F$-space.

Remarks. (1) For any space $X$ the Stone space of $A_{\Omega}(X)=\left(=Z_{\Omega}(X)\right)$ is an $F$-space.

(2) For uncountable compact metric spaces a stronger result than Theorem 5 is obtained in [1].

\section{REFERENCES}

1. F. K. Dashiell, Isomorphism problems for the Baire classes, Pacific J. Math. (to appear).

2. Z. Frolik, Realcompactness is a Baire-measurable property, Bull. Acad. Polon. Sci. Sér. Sci. Math. Astronom. Phys. 19 (1971), 617-621. MR 46 \#312. 
3. H. Gordon, Rings of functions determined by zero sets, Pacific J. Math. 36 (1971), 133-157.

4. F. Hausdorff, Set theory, Chelsea, New York, 1957. MR 19, 111.

5. J. E. Jayne, Spaces of Baire functions, Baire classes, and Souslin sets, Ph. D. Dissertation, Columbia Univ., New York, 1971.

6. - Spaces of Baire functions. I, Ann. Inst. Fourier (Grenoble) (to appear).

7. K. Kuratowski, Topology. Vol. I, Academic Press, New York, 1966.

DEPARTMENT OF MATHEMATICS, UNIVERSITY COLLEGE, LONDON, ENGLAND (Current address)

INSTITUTE OF MATHEMATICS, CZECHOSLOVAK ACADEMY OF SCIENCES, PRAGUE, CZECHOSLOVAKIA 